\title{
Estudo P.O.I.S. (prevenção da osteoporose nos idosos)
}

Ana Nunes*, Orquídea Loureiro**, Teresa Donat***, Vasyl Skorobohach****

\section{RESUMO}

Introdução: Em Portugal existem mais de 500.000 pessoas com osteoporose (OP). Procurando diminuir a morbimortalidade associada, a Direcção-Geral da Saúde e outras entidades científicas recomendam absorciometria radiológica de dupla energia (DEXA) nas mulheres > 65 anos e nos homens > 70 anos, e suplementação com cálcio 1000-1200 mg/dia e vitamina D 700$-800 \mathrm{UI} /$ dia nas pessoas $>65$ anos.

Objectivo: Avaliar e garantir a qualidade da prescrição de cálcio e vitamina $\mathrm{D}$ (Ca-VitD) e da requisição de DEXA, na prevenção da OP no idoso.

Tipo de estudo: Descritivo e transversal.

Local: Quatro centros de saúde (CS) da Região Norte.

População: Idosos inscritos nos CS estudados.

Métodos: Dimensão estudada: qualidade técnico-científica. Unidade de estudo: mulheres com 66-70 anos e homens com 71-

-75 anos, totalizando 400 idosos inscritos nos CS dos autores. Profissionais avaliados: 50 médicos de família. Período de tempo: 01.01.2006 até 30.04.2009. Tipo de dados: processo. Fonte de dados: SAM. Avaliação: interna. Critérios: 1. Prescrição de CaVitD nas mulheres; 2. Prescrição de Ca-VitD nos homens; 3. Requisição de DEXA nas mulheres; 4. Requisição de DEXA nos homens. Colheita dos dados: Maio 2009. Avaliação temporal: retrospectiva. Amostra: de base institucional. Tipo de intervenção: educacional.

Resultados: Segundo os padrões de qualidade adoptados, o critério 1 foi bom no CS Chaves-I e Ermesinde e suficiente no CS Leça da Palmeira e Unidade de Saúde Familiar (USF) Oceanos. O critério 2 foi insuficiente nos quatro CS. O critério 3 foi bom no CS Chaves-I e Leça da Palmeira e suficiente no CS Ermesinde e USF Oceanos. O critério 4 foi insuficiente nos quatro CS.

Conclusões: $O$ desconhecimento das recentes recomendações e a maior implementação das medidas de prevenção da OP nas mulheres podem explicar estes resultados. Propõe-se reavaliação após introdução de medidas correctoras, com realização de reuniões e divulgação de normas sobre o tema.

Palavras-Chave: Osteoporose; Absorciometria de raios X; Cálcio; Vitamina D; Garantia da Qualidade dos Cuidados de Saúde.

\section{INTRODUÇÃO}

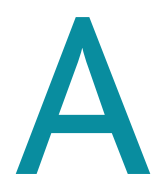
osteoporose (OP) é uma doença esquelética sistémica, caracterizada pela diminuição da massa óssea e deterioração da qualidade microestrutural do osso, que levam a uma diminuição da resistência óssea e consequente aumento do risco de fracturas.

É uma doença de elevada prevalência nos países ocidentais, sendo que em Portugal existem mais de meio

\footnotetext{
* Interna de Medicina Geral e Familiar na USF Oceanos - Centro de Saúde de Matosinhos - Unidade Local de Saúde de Matosinhos, EPE

** Interna de Medicina Geral e Familiar no Centro de Saúde de Ermesinde

*** Interna de Medicina Geral e Familiar no Centro de Saúde de Leça da Palmeira

- Unidade Local de Saúde de Matosinhos, EPE

**** Interno de Medicina Geral e Familiar no Centro de Saúde de Chaves I
}

milhão de pessoas com $\mathrm{OP}^{1,2}$. Uma em cada três mulheres e um em cada oito homens, com mais de 50 anos, são afectados pela OP. ${ }^{2,3}$ No ano 2006, no nosso país, ocorreram 2640 fracturas vertebrais e 9523 fracturas do colo do fémur. ${ }^{1,2} \mathrm{~A}$ OP é uma importante causa de morbimortalidade nos idosos, que tende a assumir proporções alarmantes com o envelhecimento da população. Os doentes com fractura do fémur proximal têm uma mortalidade entre $20-30 \%$ no ano seguinte à fractura. ${ }^{2,3}$ A OP constitui causa relevante de incapacidade na população idosa, sendo que apenas $15 \%$ dos doentes com fractura do colo femoral recuperam a capacidade funcional prévia e $40 \%$ dos doentes ficam com incapacidade grave. ${ }^{2,3}$

No idoso, a ingestão de cálcio e vitamina $\mathrm{D}$ na dieta 
diminui, havendo também um decréscimo na produção endógena de calcitriol. Com o envelhecimento ocorre redução da absorção intestinal de cálcio e da reabsorção tubular renal. A deficiência dos níveis plasmáticos de vitamina $D$ leva ao aumento da secreção de paratormona, originando perda de massa óssea excessiva e risco aumentado de fracturas.

No contexto do movimento internacional «Década do Osso e da Articulação 2000-2010», a Direcção-Geral da Saúde (DGS) tem dado a sua contribuição através da elaboração do Programa Nacional Contra as Doenças Reumáticas, ${ }^{3}$ e mais recentemente com a divulgação de normas para a utilização da absorciometria radiológica de dupla energia (DEXA) e suplementação com cálcio e vitamina $\mathrm{D}$ em pessoas idosas. ${ }^{1,2}$

A DGS e a Sociedade Portuguesa de Reumatologia preconizam a realização de DEXA nas mulheres com idade superior a 65 anos e nos homens com idade superior a 70 anos, recomendações que são apoiadas pela UPSTF e outras entidades científicas.,4-7

Nos indivíduos com mais de 65 anos, com primeira DEXA normal, não é necessária a repetição do exame. De um modo geral, nos doentes osteoporóticos pode-se repetir a DEXA após mais de dois anos de terapêutica, enquanto que nos doentes com osteopenia pode ser repetida depois de três a cinco anos.,4,6

A vitamina $\mathrm{D}$ tem um papel importante na manutenção dos níveis séricos de cálcio. No entanto, a síntese cutânea desta vitamina está dependente de vários factores, como latitude da zona geográfica, estação do ano, hora do dia, a superfície corporal exposta ao sol e duração da exposição, uso de cremes com protecção solar, pigmentação da pele, obesidade e idade. Para além disso, o aporte de cálcio na alimentação da população Portuguesa é frequentemente insuficiente. As razões expostas justificam a importância da suplementação com cálcio e vitamina D na prevenção da $\mathrm{OP}$.

De acordo com a DGS, as pessoas com mais de 65 anos têm indicação para realizar suplementação com cálcio nas doses de 1000 a 1200 mg diárias e vitamina D na dosagem de 700 a 800 UI por dia. ${ }^{1}$ A idade superior a 65 anos é considerada factor de risco major para a OP.

Também na declaração Portuguesa da Vitamina D, documento certificado pela Associação Portuguesa dos Médicos de Clínica Geral, Sociedade Portuguesa de
Doenças Ósseas Metabólicas, Sociedade Portuguesa de Endocrinologia, Diabetes e Metabolismo e Sociedade Portuguesa de Ortopedia e Traumatologia, entre outras, é aconselhada a suplementação com vitamina D, em doses de pelo menos 700 a 800 UI por dia, para redução do risco de fracturas. ${ }^{8}$

Consensos internacionais da National Osteoporosis Foundation (NOF) e da International Osteoporosis Foundation (IOF), também suportam a suplementação diária com cálcio e vitamina D. ${ }^{8,9,10}$

A OP é um importante problema de saúde pública. O investimento na área da prevenção da OP é de fulcral relevância ao nível dos Cuidados de Saúde Primários, não só pela elevada prevalência desta doença na população idosa, mas principalmente pelo impacto na morbimortalidade e qualidade de vida, não esquecendo os encargos sociais e económicos. Deste modo, é essencial adoptar estratégias de prevenção custo-efectivas, que incluem a suplementação com cálcio e vitamina $D$ e a requisição de DEXA.

A ideia de realizar este trabalho de garantia da qualidade surgiu decorrente da percepção dos autores de que a prevenção da osteoporose nos idosos não é realizada de forma adequada.

\section{OBJECTIVO}

É objectivo deste estudo avaliar e garantir a qualidade da prescrição de cálcio e vitamina $\mathrm{D}$ e da requisição de DEXA, como medidas de prevenção da osteoporose no idoso.

\section{MÉTODOS}

A dimensão estudada foi a qualidade técnico-científica dos registos dos médicos de família na prevenção da osteoporose no idoso.

A população estudada incluiu idosos do sexo feminino entre os 66 e os 70 anos de idade (nascidas entre 1.05.1938 e 30.04.1943) e do sexo masculino entre os 71 e os 75 anos de idade (nascidos entre 1.05.1933 e 30.04.1938), inscritos em médico de família nos centros de saúde onde os autores do estudo exercem a sua actividade (CS Matosinhos/USF Oceanos, CS Leça da Palmeira, CS Ermesinde e CS de Chaves I).

A amostra foi de base institucional (centros de saúde). Foram obtidas listagens de todos os idosos inscritos com idades compreendidas entre as faixas etárias 
descritas, através do programa informático SINUS. Destes, foram seleccionados 100 utentes ( 50 mulheres e 50 homens) por cada centro de saúde, de forma aleatória com reposição, obtendo-se uma dimensão amostral de 400 idosos.

Os profissionais avaliados foram todos os médicos de família dos CS mencionados anteriormente, num total de 50 médicos. O período de tempo que se avaliou foi de 01.01.2006 até 30.04.2009.

O tipo de dados analisados foi de processo.

A fonte de dados correspondeu ao processo do utente em suporte informático - SAM (Sistema de Apoio ao Médico) versão 9.0, com pesquisa na área de prescrição de receituário e na área de requisição de meios complementares de diagnóstico.

O tipo de avaliação foi interna.

Consideraram-se os seguintes critérios e padrões de qualidade:

Critério 1: Pelo menos um registo de prescrição de cálcio e vitamina D nas mulheres com 66-70 anos

Padrão de qualidade adoptado para o critério 1 :

- Bom > 30\%

- Suficiente $10-30 \%$

- Insuficiente $<10 \%$

Critério 2: Pelo menos um registo de prescrição de cálcio e vitamina D nos homens com 71-75 anos

Padrão de qualidade adoptado para o critério 2:

- Bom $>30 \%$

- Suficiente $10-30 \%$

- Insuficiente $<10 \%$

Critério 3: Pelo menos um registo de requisição de DEXA nas mulheres com 66-70 anos

Padrão de qualidade adoptado para o critério 3 :

- Bom $>40 \%$

- Suficiente $20-40 \%$

- Insuficiente $<20 \%$

Critério 4: Pelo menos um registo de requisição de DEXA nos homens com 71-75 anos

Padrão de qualidade adoptado para o critério 4:

- Bom $>30 \%$

- Suficiente $10-30 \%$

- Insuficiente $<10 \%$

A colheita dos dados foi realiza- da pelos autores do estudo, durante o mês de Maio de 2009.

A avaliação foi retrospectiva.

O tipo de intervenção prevista é de base educacional, de forma a implementar a requisição de DEXA e a prescrição de cálcio e vitamina $D$ nas faixas etárias adequadas, como medida de prevenção da osteoporose integrada nos cuidados à população idosa.

\section{RESULTADOS}

Foram analisados os registos dos pedidos de DEXA e prescrição de cálcio e vitamina $D$ num total de 400 utentes, 100 utentes por cada CS estudado.

Em relação ao padrão de qualidade obtido para os critérios 1 e 3 no CS Chaves I verificou-se que é bom (Figura 1 e 3). O padrão de qualidade dos critérios 2 e 4 é insuficiente (Figuras 2 e 4).

No CS Ermesinde o padrão de qualidade verificado para o critério 1 é bom, para o critério 3 é suficiente, sendo o padrão de qualidade dos critérios 2 e 4 insuficiente (Figuras 1 a 4).

No CS Leça da Palmeira o padrão de qualidade do critério 1 é suficiente, do critério 3 é bom e dos critérios 2 e 4 é insuficiente (Figuras 1 a 4).

Na USF Oceanos o padrão de qualidade dos critérios 1 e 3 é suficiente e dos critérios 2 e 4 é insuficiente (Figuras 1 a 4 ).

No total dos quatro CS avaliados o padrão de qualidade dos critérios 1 e 3 é bom. Relativamente aos critérios 2 e 4 o padrão é insuficiente (Figura 5).

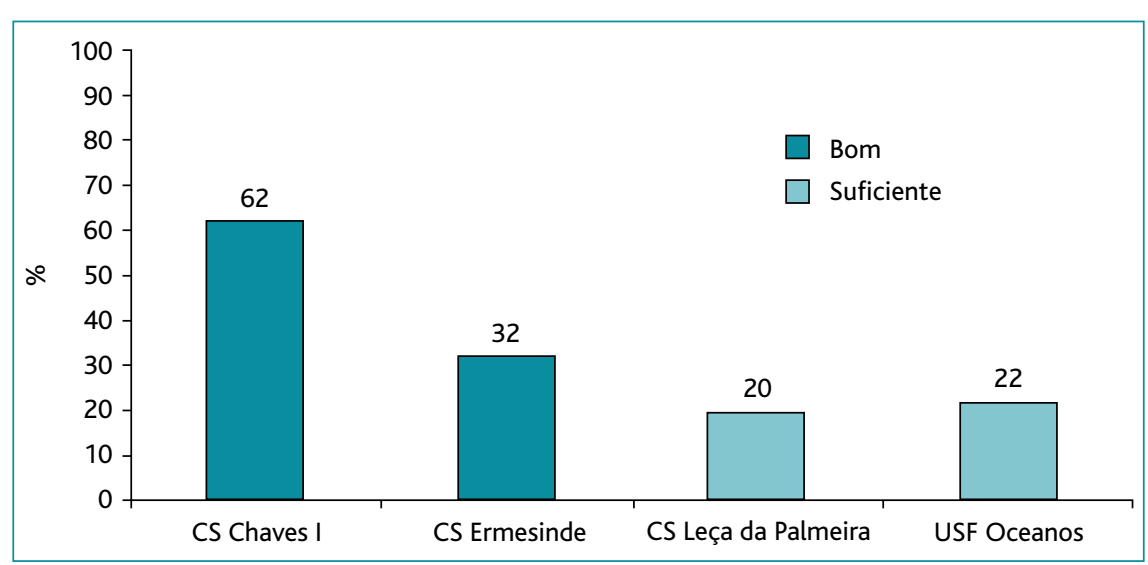

Figura 1. Critério 1 - Prescrição de cálcio e vitamina D nas mulheres. 


\section{DISCUSSÃO}

De acordo com os resultados obtidos, verifica-se que o critério 1 (pelo menos um registo de prescrição de cálcio e vitamina D nas mulheres com 66-70 anos) atinge um padrão de qualidade bom no CS Chaves I e CS Ermesinde e suficiente no CS Leça da Palmeira e USF Oceanos.

Para o critério 2 (pelo menos um registo de prescrição de cálcio e vitamina D nos homens com 71-75 anos), obteve-se um padrão de qualidade insuficiente em todos os CS. O resultado obtido para este critério pode reflectir um desconhecimento por parte dos profissionais das recentes recomendações da DGS, que aconselham a suplementação com cálcio e vitamina $\mathrm{D}$ a todas as pessoas com mais de 65 anos de idade.

Nos resultados, o critério 3 (pelo menos um registo de requisição de DEXA nas mulheres com 66-70 anos) obteve um padrão de qualidade bom nos CS Chaves I e Leça da Palmeira e suficiente no CS Ermesinde e na USF Oceanos. Para o critério 4 (pelo menos um registo de requisição de DEXA nos homens com 71-75 anos) o padrão de qualidade foi insuficiente para todos os CS. Tal como seria de prever, a requisição de DEXA nas mulheres está muito mais integrada na prática clínica em comparação com a requisição nos homens. Uma possível explicação para este facto é que as normas e guidelines anteriores sempre estiveram mais orientadas para as mulheres.

Numa análise geral dos resultados, podemos observar que as medidas de prevenção da OP nos idosos estão mais implementadas nas mulheres do que nos ho-

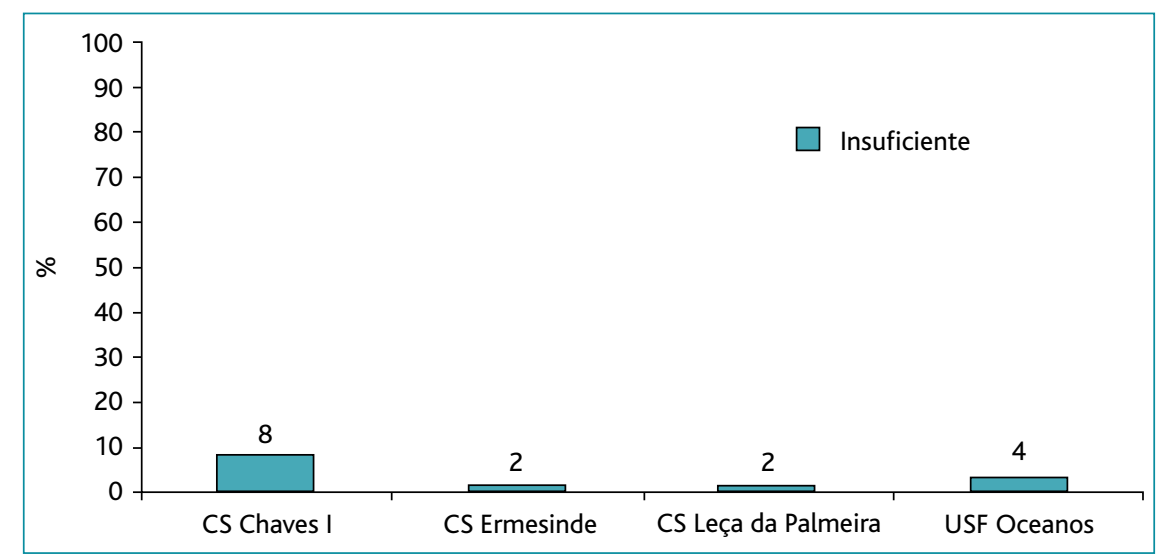

Figura 2. Critério 2 - Prescrição de cálcio e vitamina D nos homens.

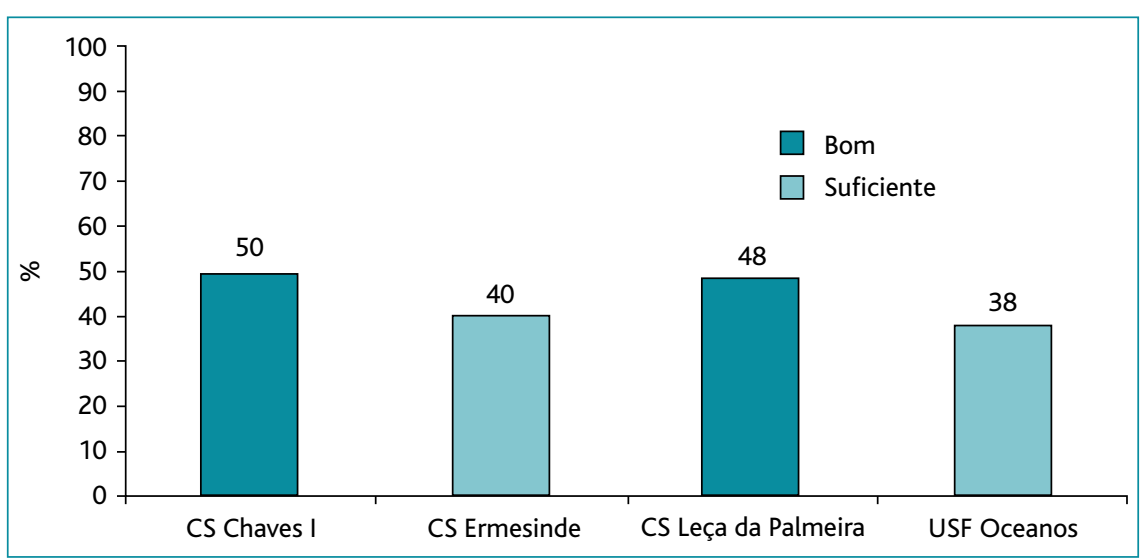

Figura 3. Critério 3 - Requisição de DEXA nas mulheres.

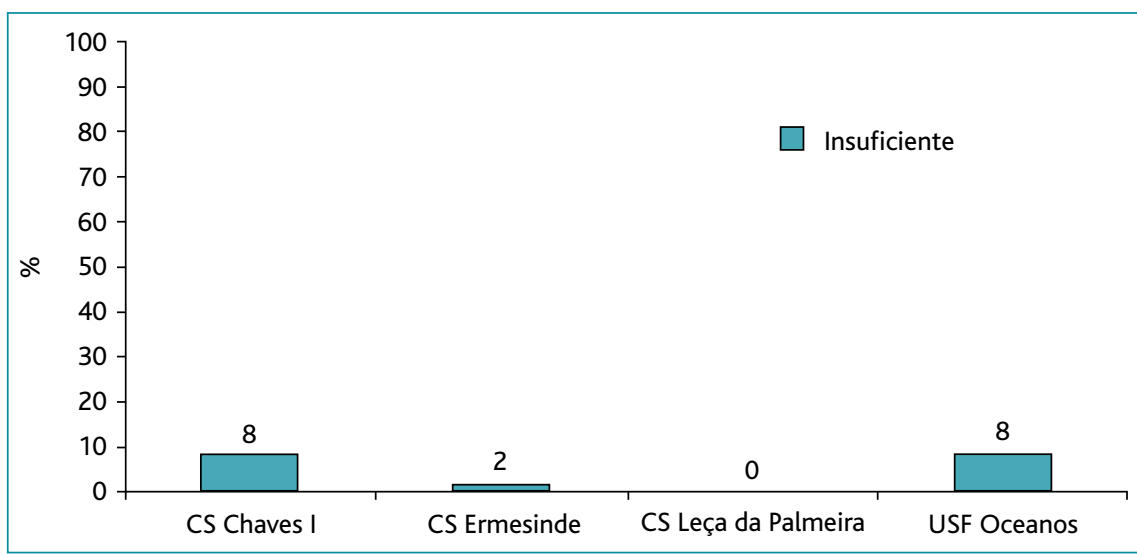

Figura 4. Critério 4 - Requisição de DEXA nos homens

mens. Existe ainda um importante caminho a percorrer no sentido de melhorar a prescrição de cálcio e vitamina D e a requisição de DEXA nos idosos do sexo 


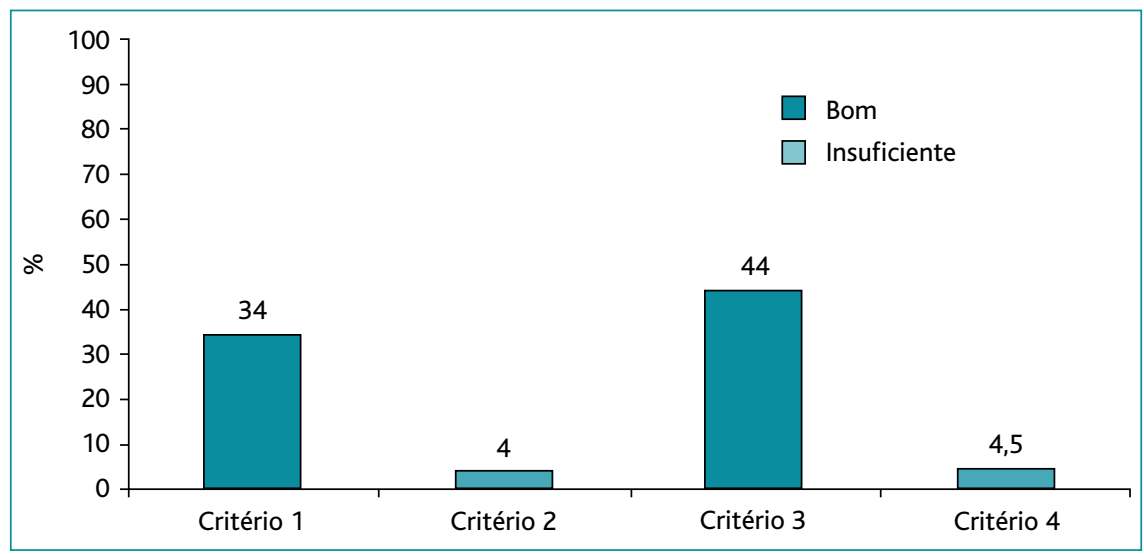

Figura 5. Avaliação global de todos os CS por critério.

masculino, tornando-se fulcral uma melhoria contínua da qualidade.

De ressalvar que neste estudo foram apenas avaliados os registos informáticos do SAM, que se encontra em pleno funcionamento nos CS dos autores apenas desde 2006. Este aspecto pode ter originado uma subvalorização dos resultados obtidos, que os autores tentaram minimizar ao máximo através da imposição de limites nas faixas etárias da população de idosos avaliada.

A avaliação de quatro CS com realidades distintas, quer ao nível da população inscrita quer em funcionamento e organização, bem com o número elevado de médicos avaliados neste estudo, constituem alguns dos pontos fortes deste trabalho.

\section{MEDIDAS CORRECTORAS}

Como forma de melhorar e garantir a qualidade da requisição de DEXA e prescrição de cálcio e vitamina D e dada a importância do tema OP na Medicina Geral e Familiar, propõe-se a implementação de varias medidas correctoras:

\section{Medidas de análise}

1. Analisar as medidas implementadas nos CS que obtiveram padrão de qualidade bom, com o propósito de reproduzir e alargar essas atitudes aos restantes CS deste estudo.

\section{Medidas formativas}

2. Realizar reuniões científicas nos CS participantes, com vista a sensibilizar os médicos de família para a importância da requisição de DEXA e prescrição de cálcio e vitamina $\mathrm{D}$ como medidas de prevenção da osteoporose. Dar a conhecer a situação actual relativamente aos registos neste âmbito através da apresentação deste estudo. A reunião será agendada com os directores dos CS e coordenador da USF e divulgada atempadamente em reunião de serviço a todos os médicos. A organização das reuniões, bem como as comunicações orais, estarão a cargo dos próprios autores deste estudo.

\section{Medidas informativas}

3. Divulgar as normas da DGS de 2008 sobre o tema e criar métodos para facilitar o acesso dos médicos a todas as circulares emitidas, através da criação de dossiers na biblioteca dos CS actualizados periodicamente pelos internos em formação. Introduzir pastas nos computadores de cada consultório médico, na área do ambiente de trabalho, contendo as referidas normas. Destaque para a orientação técnica sobre suplemento de cálcio e vitamina $\mathrm{D}$ em pessoas idosas e para a orientação técnica relativa à utilização da absorciometria radiológica de dupla energia.

Medidas de execução

4. Desenvolver um protocolo de vigilância do idoso e prevenção da osteoporose, elaborado pelos internos dos CS envolvidos neste estudo, que deverá funcionar como um manual de boas práticas para os médicos.

Medidas de intervenção

5. Propor aos directores dos CS e coordenador da USF a avaliação destes parâmetros nos planos de acção desenvolvidos em cada unidade, de forma a incentivar a sua realização. Reunir com o conselho técnico, para definir estratégias de actuação conjuntas, de

\section{QUADRO I. Cronograma e calendário de reavaliação}

\begin{tabular}{l|l} 
Período & Actividades \\
\hline $2^{\circ}$ Semestre de 2009 & $\begin{array}{l}\text { Implementação das medidas } \\
\text { correctoras }\end{array}$ \\
\hline Maio de 2010 & Reavaliação da qualidade
\end{tabular}


forma a implementar as recomendações da DGS sobre a prevenção da OP.

6. Contactar o Instituto de Gestão Informática e Financeira da Saúde, propondo a criação de um perfil de avaliação do idoso no programa informático SAM, como um programa de saúde independente, com local específico para o registo da requisição de DEXA e respectivo resultado (normal / osteopenia / osteoporose), bem como para o registo da prescrição de cálcio e vitamina D (sim / não).

Com estas medidas correctoras, os autores do estudo pretendem um aumento do padrão de qualidade dos critérios 1 e 3 para bom e dos critérios 2 e 4 para pelo menos suficiente em todos os CS, até Maio de 2010, altura em que se realizará nova avaliação da qualidade.

A melhoria da qualidade dos registos é um processo contínuo que requer tempo para a sua implementação. Segue-se o cronograma e calendário de reavaliação propostos, com o planeamento das actividades a desenvolver, para o desenrolar deste programa para o desenvolvimento contínuo da garantia da qualidade da requisição de DEXA e prescrição de cálcio e vitamina D (Quadro I).

\section{REFERÊNCIAS BIBLIOGRÁFICAS}

1. Direcção-Geral da Saúde; Divisão de Prevenção e Controlo da Doença / Direcção de Serviços da Qualidade Clínica. Orientação técnica sobre suplemento de cálcio e vitamina D em pessoas idosas. Lisboa: DGS; 2008.

2. Direcção-Geral da Saúde; Divisão de Prevenção e Controlo da Doença / Direcção de Serviços da Qualidade Clínica. Orientação técnica para a utilização da absorsiometria radiológica de dupla energia (DEXA). Lisboa: DGS; 2008.

3. Direcção-Geral da Saúde; Divisão das Doenças Genéticas, Crónicas e Geriátricas. Programa Nacional Contra as Doenças Reumáticas. Lisboa: DGS; 2004.

4. Tavares V, Canhão H, Gomes JA, Simões E, Romeu JC, Coelho P, et al.
Recomendações para o diagnóstico e terapêutica da osteoporose. Acta Reumatol Port 2007 Jan-Mar; 32 (1): 49-59.

5. U.S. Preventive Services Task Force. Screening for osteoporosis in postmenopausal woman: recommendations and rationale. Rockville, MD: Agency for Healthcare Research and Quality; September 2002. Disponível em: http://www.ahrq.gov/clinic/3rduspstf/osteoporosis/osteorr.htm [acedido em 20/06/2009].

6. Neff MJ; American College of Obstetricians and Gynecologists. ACOG Releases Guidelines for Clinical Management of Osteoporosis. Am Fam Physician 2004 Mar 15; 69 (6): 1558-60.

7. Baim S, Binkley N, Bilezikian JP, Kendler DL, Hans DB, Lewiecki EM, et al. Official positions of the International Society for Clinical Densitometry and executive summary of the 2007 ISCD Position Development Conference. J Clin Densitom 2008 Jan-Mar; 11 (1): 75-91.

8. Associação Portuguesa dos Médicos de Clínica Geral, Sociedade Portuguesa de Doenças Ósseas Metabólicas, Sociedade Portuguesa de Endocrinologia, Diabetes e Metabolismo, Sociedade Portuguesa de Ortopedia e Traumatologia. Declaração Portuguesa da Vitamina D. 2009.

9. National Osteoporosis Foundation (NOF). National Osteoporosis Foundation's Updated recommendations for calcium and vitamin D3 intake. Disponível em http://www.nof.org.prevention/calcium_and_vitaminD.htm. [acedido em 20/06/2009]

10. Vega CP. A supplementation with calcium and vitamin D: efficacy against fracture and total mortality: a best evidence review. MedscapeCME. Publicado em 2.05.2008. [acedido em 20/06/2009].

\section{AGRADECIMENTOS}

Os autores desejam agradecer à Dr. ${ }^{a}$ Ana Sardinha e à Dr. ${ }^{a}$ Emília Teixeira pelos conhecimentos e apoio prestados durante a realização deste estudo, no âmbito do Curso de Garantia da Qualidade em Cuidados de Saúde Primários da Coordenação do Internato de Medicina Geral e Familiar da Zona Norte.

\section{ENDEREÇO PARA CORRESPONDÊNCIA}

Ana Filipa Coelho Nunes

USF Oceanos - Centro de Saúde de Matosinhos

Rua Alfredo Cunha, n. ${ }^{\circ}$ 365, 4450-021 Matosinhos

E-mail: anafcnunes@hotmail.com

Recebido em 12/10/2009

Aceite para publicação em 09/02/2010 


\section{ABSTRACT}

\section{STUDY OF OSTEOPOROSIS PREVENTION IN THE ELDERLY}

Introduction: In Portugal there are more than 500,000 people with osteoporosis (OP). In order to reduce the associated morbimortality, the Directorate General of Health and other scientific organizations recommend dual-energy X-ray absorptiometry (DEXA) in women > 65 years and men > 70 years, and supplementation with calcium 1000-1200 mg/day and vitamin D $700-800 \mathrm{IU} /$ day in people $>65$ years.

Aim: Evaluate and guarantee the quality of calcium and vitamin D (Ca-VitD) prescription and of DEXA request, in the prevention of OP in elderly patients.

Type of study: Descriptive and transversal.

Place: Four primary care centers (PCC) on the Northern Region.

Population: Elderly registered on the PCC studied.

Methods: Studied dimension: technical-scientific quality. Unit of study: women aged 66-70 years and men aged 71-75 years, totalizing 400 elderly registered on the authors PCC. Evaluated professionals: 50 family doctors. Time period: 01.01 .2006 to 30.04.2009. Type of data: process. Data source: SAM. Type of evaluation: internal. Criteria: 1. Prescription of Ca-VitD in women 2. Prescription of Ca-VitD in men 3. DEXA request in women 4. DEXA request in men. Data collecting: May 2009. Temporal evaluation: retrospective. Sample: of institutional basis. Type of intervention: educational.

Results: According to the adopted quality standards, criterion 1 was good at PCC Chaves-I and Ermesinde and sufficient at PCC Leça da Palmeira and USF Oceanos. Criterion 2 was insufficient in all four PCC. Criterion 3 was good at PCC Chaves-I and Leça da Palmeira and sufficient at PCC Ermesinde and USF Oceanos. Criterion 4 was insufficient in all four PCC.

Conclusion: The unknowledge of the recent recommendations and the higher implementation of OP prevention measures in women may explain these results. It is proposed a reassessment after the introduction of corrective measures, with meetings and disclosure of guidelines on the subject.

Keywords: Osteoporosis; X-ray Absorptiometry; Calcium; Vitamin D; Health Care Quality Assurance. 